\title{
Efficient generation of functional hepatocyte- like cells from mouse liver progenitor cells via indirect co-culture with immortalized human hepatic stellate cells
}

\author{
Xiao-Ping Pan, Yi-Ni Wang, Xiao-Peng Yu, Chun-Xia Zhu, Jian-Zhou Li, Wei-Bo Du, \\ Yi-Min Zhang, Hong-Cui Cao, Yan-Hong Zhang, Dan-Hua Zhu,
} George C Yeoh and Lan-Juan Li

Hangzhou, China

\begin{abstract}
BACKGROUND: Differentiation of liver progenitor cells (LPCs) to functional hepatocytes holds great potential to develop new strategies for hepatocyte transplantation and the screening of drug-induced cytotoxicity. However, reports on the efficient and convenient hepatic differentiation of LPCs to hepatocytes are few. The present study aims to investigate the possibility of generating functional hepatocytes from LPCs in an indirect co-culture system.
\end{abstract}

METHODS: Mouse LPCs were co-cultured in Transwell plates with an immortalized human hepatic stellate cell line (HSCLi) we previously established. The morphology, expression of hepatic markers, and functions of mouse LPC-derived cells were monitored and compared with those of conventionally cultured LPCs.

RESULTS: Co-culturing with HSC-Li cells induced differentiation of mouse LPCs into functional hepatocyte-like cells. The differentiated cells were morphologically transformed into hepatocyte-like cells 3 days after co-culture initiation. In addition, the differentiated cells expressed liver-specific genes and possessed hepatic functions, including glycogen storage, lowdensity lipoprotein uptake, albumin secretion, urea synthesis, and cytochrome P450 1A2 enzymatic activity.

CONCLUSIONS: Our method, which employs indirect co-culture with HSC-Li cells, can efficiently induce the differentiation of LPCs into functional hepatocytes. This finding suggests that this co-culture system can be a useful method for the efficient generation of functional hepatocytes from LPCs.

(Hepatobiliary Pancreat Dis Int 2016;15:173-179)

KEY WORDS: liver progenitor cells; indirect co-culture; cell differentiation; hepatocytes; immortalized human hepatic stellate cells

\section{Introduction}
$A$ cute liver failure is a lethal clinical condition.
Orthotopic liver transplantation is the only de-
finitive treatment for this condition. However, a shortage of donor organs, high cost, and the requirement for lifelong immunosuppressive treatment often pre- clude liver transplantation. Hepatocyte transplantation has been evaluated in clinical trials. This procedure may provide a valuable adjuvant treatment to liver disease. However, the progress of this strategy is dramatically limited by poor proliferation capacity of primary hepa- tocytes and the paucity of donor cells. Therefore, an effi- cient and reproducible method for generating functional versity School of Medicine, Hangzhou 310003, Chin (Pan XP, Way Yu XP, Zhu CX, Li JZ, Du WB, Zhang YM, Cao HC, Zhang YH, Zhu DH and Li LJ); College of Basic Medical Sciences, Zhejiang Chinese Medical University, Hangzhou 310053, China (Pan XP); Biochemistry \& Molecular Biology Crawley, University of Western Australia, Western Australia 6009, Australia (Yeoh GC)

Corresponding Author: Lan-Juan Li, MD, Collaborative Innovation Center for Diagnosis and Treatment of Infectious Diseases; State Key Laboratory for Diagnosis and Treatment of Infectious Diseases, First Affiliated Hospital, Zhejiang University School of Medicine, Hangzhou 310003, China (Tel/Fax: +86-571-87236759; Email: 1jli@zju.edu.cn)

(C) 2016, Hepatobiliary Pancreat Dis Int. All rights reserved. doi: 10.1016/S1499-3872(16)60074-7

Published online February 24, 2016. 
hepatocytes from stem/progenitor cells can provide cells that are useful for preclinical research and drug screening and potentially to develop new therapeutic strategies for liver disease. ${ }^{[1-5]}$

Liver progenitor cells (LPCs), also called oval cells in rodents because of their morphology, exhibit longterm proliferation in vitro, while maintaining bipotential capacity to give rise to both mature hepatocytes and cholangiocytes. ${ }^{[6,7]}$ Thus, LPCs may be an attractive candidate to provide mature hepatocytes. Studies on LPC differentiation have been previously reported, but these investigations are commonly focused on the culture medium containing cytokines (i.e., hepatocyte growth factor, oncostatin $\mathrm{M}$, dexamethasone $)^{[4,8,9]}$ or gene modification (i.e., Foxa2, $\operatorname{Hnf} 4 \alpha, \mathrm{C} / \mathrm{ebp} \alpha),{ }^{[10,11]}$ which are complex and expensive. Moreover, they do not efficiently convert LPCs to hepatocytes.

The effect of niche cells on stem/progenitor cells in differentiation has been demonstrated in several tissues, ${ }^{[12-16]}$ which lays the foundation in building a coculture system to mimic the internal microenvironment in vitro. A previous study ${ }^{[17]}$ proved that paracrine signals produced by different feeder cells governed the differentiation fates of hepatic stem cells. Researchers applied coculture methods to promote hepatic differentiation from embryonic stem cells, ${ }^{[3,18,19]}$ induced pluripotent stem cells, ${ }^{[20]}$ or mesenchymal stem cells (MSCs). ${ }^{[21]}$ However, an efficient co-culture method for hepatocyte differentiation of LPCs has not been established.

Hepatic stellate cells (HSCs) is one of the major hepatic nonparenchymal cells, which plays a pivotal role in liver immunity and liver fibrogenesis. Fully activated HSCs have been recently demonstrated to induce the differentiation of MSCs and nonhepatocytic epithelial stemlike cells into hepatocyte-like cells through secreting prohepatocyte differentiation factors. ${ }^{[21,22]}$ We have previously established an immortalized human HSC line (HSC-Li), which is fully activated. ${ }^{[23]}$ We hypothesized that HSC-Li cells may promote the differentiation of LPCs into hepatocytes. To test this hypothesis, we developed an indirect co-culture system of mouse LPCs and HSC-Li cells and evaluated the morphologic, phenotypic, and functional characteristics of LPC-derived differentiated cells.

\section{Methods}

\section{Animals}

Male C57BL/6J mice (9 weeks) were obtained from B\&K Universal Group Limited (Shanghai, China). All animal experiments were approved by the ethics committee of the First Affiliated Hospital, Zhejiang University School of Medicine, and performed in accordance with the In- terdisciplinary Principles and Guidelines for the Use of Animals in Research, Testing, and Education by the New York Academy of Sciences, Ad Hoc Animal Research Committee. Surgery to obtain mouse primary hepatocytes was performed under sodium pentobarbital anesthesia.

\section{Culture of mouse LPCs and HSC-Li}

HSC-Li cell line was established as described previously. ${ }^{[23]}$ BMEL-TAT, a mouse LPC line, was derived from a TAT-GRE lacZ transgenic mouse placed on a choline deficient, ethionine supplemented diet. ${ }^{[24,25]}$ Hepatic differentiation of BMEL-TAT cells was assessed and quantified by measuring the expression of lac $Z$ transgene, which is regulated by TAT promoter (a marker of adult hepatocytes). HSC-Li and BMEL-TAT cells were cultured in Dulbecco's Modified Eagle Medium (DMEM) containing $10 \%$ fetal bovine serum (FBS). Culture medium was replaced every 2 to 3 days. When cultures reached $80 \%$ confluence, the cells were trypsinized with $0.05 \%$ trypsin-EDTA and re-plated.

\section{Proteome array of HSC-Li cell conditioned medium}

Conditioned medium was prepared by collecting serum-free medium after culturing HSC-Li cells for 24 hours. The levels of specific cytokines in conditioned medium were analyzed with proteome profiler antibody arrays that included human growth factor antibody array and human inflammation antibody array (Products code: AAH-GF-1-4 and AAH-INF-3-4, respectively, Ray Biotech, USA), according to manufacturer instructions. Human growth factor antibody array includes 41 growth factors, and human inflammation antibody array includes 40 inflammatory factors. Serum-free DMEM was used as a control.

\section{Co-culture of mouse LPCs with HSC-Li}

HSC-Li and BMEL-TAT cells, in logarithmic growth phase, were seeded in Transwell plates (pore size: $3 \mu \mathrm{m}$; Millipore, USA) at a ratio of 1:2. BMEL-TAT cells (2.0× $\left.10^{5}\right)$ and HSC-Li cells $\left(1.0 \times 10^{5}\right)$ were inoculated into the lower and upper chambers of Transwell plates, respectively. Mono-cultured BMEL-TAT cells served as controls. Cells from both groups were maintained in DMEM containing 10\% FBS. The morphology, gene expression, hepatocytic markers, and functions in the two groups were examined after co-culture at different time.

\section{Preparation of mouse primary hepatocytes}

Mouse primary hepatocytes served as positive controls and were isolated from male C57BL/6J mouse liver by two-step collagenase digestion as previously described. ${ }^{[26]}$ Mouse liver was perfused with ethylene 
glycol-bis (2-aminoethylether)-N, N, N', N'-tetraacetic acid $(0.5 \mathrm{mmol} / \mathrm{L})$ and collagenase IV in Hank's balanced salt solution without $\mathrm{CaCl}_{2}$. Suspended hepatocytes were washed and collected by centrifugation at $4{ }^{\circ} \mathrm{C}$ twice. After discarding the supernatant, cell viability was determined by trypan blue exclusion. Hepatocytes at a cell density of $2.0 \times 10^{5}$ in $2 \mathrm{~mL}$ DMEM medium containing $10 \%$ FBS were seeded in 6-well polystyrene plates.

\section{Reverse-transcription PCR (RT-PCR) and real-time RT-PCR assay}

Total RNA was extracted using the RNeasy plus Mini Kit (Qiagen, USA) and cDNA prepared using a reverse transcription kit (A3800; Promega, USA) according to the manufacturer instruction. PCR was conducted with a PCR Thermocycle Instrument (PTC200, Bio-Rad, USA) using 40 cycles at $95{ }^{\circ} \mathrm{C}$ for 10 seconds, $94{ }^{\circ} \mathrm{C}$ for 5 seconds, and $60{ }^{\circ} \mathrm{C}$ for 35 seconds. The specific primers for albumin, cytokeratin 18 (CK18), tyrosine aminotransferase (TAT), and glucose-6-phoshpatase (G6P) are described in a previous study. ${ }^{[3]}$ Glyceraldehyde-3-phosphate dehydrogenase (GAPDH) was used as a reference.

\section{Periodic acid Schiff (PAS) staining}

Glycogen accumulated in cultured cells was revealed using a PAS kit (395B-1KT, Sigma, USA) and viewed and recorded under brightfield illumination.

\section{$\beta$-galactosidase cytochemical assay}

$\beta$-galactoside reacted with $\mathrm{X}$-gal was measured with an in situ $\beta$-galactosidase staining kit (Beyotime, China) to determine the expression of the lacZ gene in BMELTAT cells. The cultured cells were incubated with X-gal for 24 hours according to the manufacturer's instructions. Then the stained cells were observed and recorded with a microscope (Olympus, Japan).

\section{Immunofluorescence staining}

Cells were fixed with $4 \%$ paraformaldehyde for 15 minutes, permeabilized with $0.2 \%$ Triton X-100, and blocked with $10 \%$ bovine serum, followed by incubation with rabbit anti-mouse AFP, albumin (Santa Cruz, USA), rabbit anti-CK18 monoclonal antibody (Thermo Fisher Scientific, USA), or rabbit anti-mouse CK19 antibody (Abcam, USA) for 60 minutes. After washing, the cells were incubated with secondary antibody for 30 minutes. The secondary antibodies include donkey anti-rabbit IgG FITC (Abcam) and goat anti-rabbit IgG Dylight 650 (Abcam). The presence of proteins of interest was examined and recorded with a fluorescence microscope (IX81, Olympus).

\section{Low-density lipoprotein (LDL) uptake assay}

The cells were incubated with 3, 3'-dioctadecylindocar bocyanine low-density lipoprotein (DiI-LDL, $10 \mu \mathrm{g} / \mathrm{mL}$, Sigma) in the cell culture incubator for 24 hours to assess their ability to absorb LDL. LDL uptake in cultured cells was observed and recorded with a fluorescence microscope (IX81, Olympus).

\section{Urea synthesis and albumin secretion}

Culture supernatants were collected to quantify urea production with a QuantiChrom Urea Assay Kit (Sigma) according to the manufacturer's instructions. The level of mouse albumin in cell supernatants was measured with an Albumin Mouse ELISA Kit (Abcam).

\section{Cytochrome $\mathbf{P} 450$ activity assay}

The enzymatic activity of cytochrome 1A2 (CYP1A2) was determined by the P450-Glo CYP1A2 Assay (Promega). Luminescence was recorded with a multimode reader (DTX880; Beckman Coulter, USA). The assay was performed as described previously. ${ }^{[27]}$

\section{Statistical analysis}

All data were presented as mean \pm SD. Statistical analyses were based on a paired $t$ test using SPSS 15.0, and A $P<0.05$ was considered statistically significant.

\section{Results}

\section{Characterization of HSC-Li conditioned medium}

Conditioned medium derived from HSC-Li was examined with proteome profiler human arrays specific for cytokines and growth factors. HSC-Li cells produced the following growth factors that are responsible for hepatocyte protection: epidermal growth factor (EGF), plateletderived growth factor-BB, vascular endothelial growth factor-D (VEGF-D), insulin-like growth factor-1, and hepatocyte growth factor (HGF). In addition to growth factors, inflammatory factors were also released, such as intercellular cell adhesion molecule-1, interferon-gamma, interleukin-6 (IL-6), transforming growth factor- $\beta$, and tumor necrosis factor- $\alpha$ (TNF- $\alpha$ ). We classified and analyzed these cytokines according to functions to gain systemic understanding about cytokines produced by HSCLi cells. The results showed that cytokines can be divided into six classes (Fig. 1): hepatocyte protection, hepatocyte growth, anti-apoptosis, liver-specific metabolic activity enhancement, anti-inflammation, and angiogenesis.

\section{Phenotype of differentiated LPCs}

To establish a co-culture system, BMEL-TAT cells 
were seeded in the lower layer of Transwell plates, and HSC-Li cells in the upper layer. Mono-cultured BMELTAT cells were used as controls (Fig. 2A). The morphology of the BMEL-TAT cells showed remarkable changes during culture (Fig. 2B-F). They acquired hepatic morphology, and increased cell size after co-culture for 3 days, in contrast to the initial small cells that are phenotypically immature with scant cytoplasm and oval-shaped nuclei. RT-PCR analysis that included hepatocyte-specific genes (albumin, CK18, TAT, and G6P) was performed to determine the degree of hepatic differentiation during the culture process. Expression levels of all these genes were gradually increased over the course of the differentiation process (Fig. 3A). Expression of $\beta$-galactosidase under the regulation of the TAT gene that is only expressed in mature hepatocytes was also increased. Staining of LPCs with X-gal was only observed after coculture of BMEL-TAT cells with HSC-Li cells for 21 days
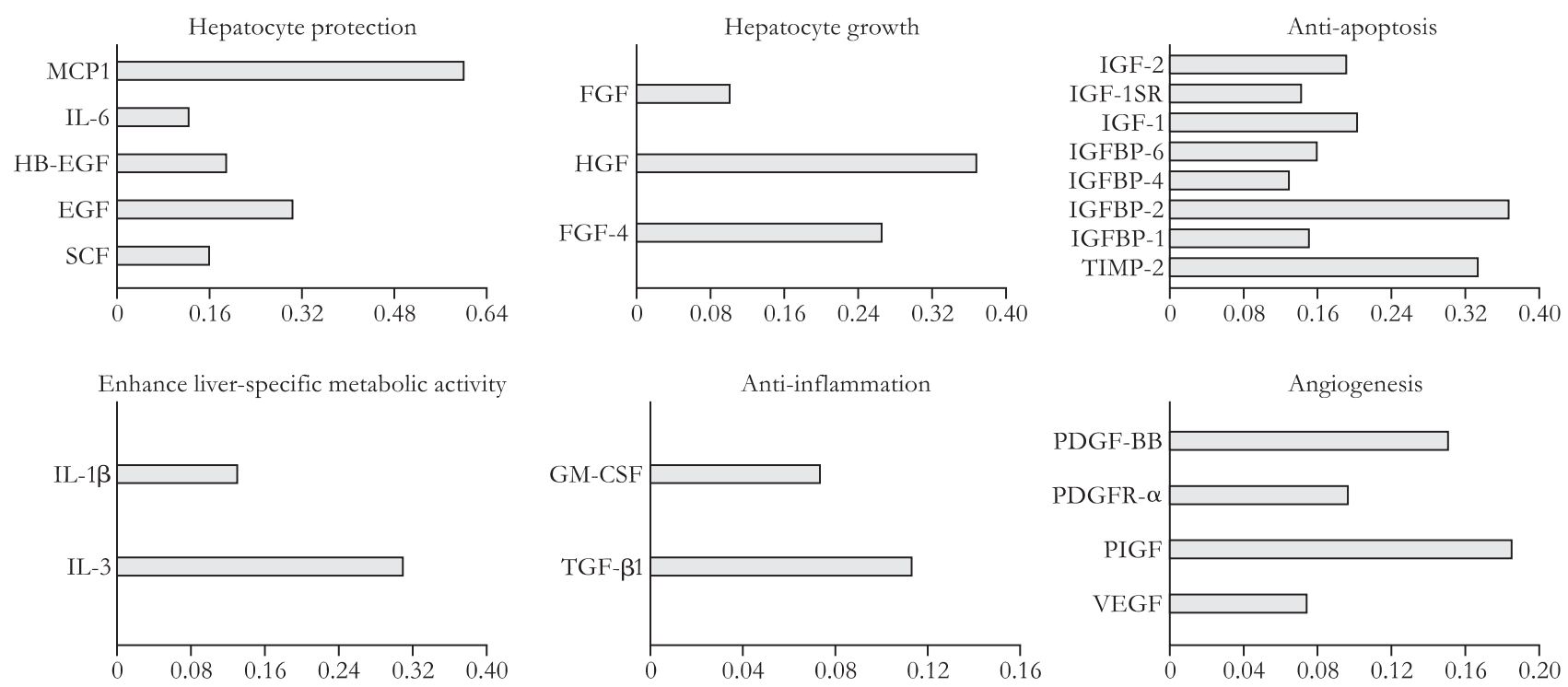

Fig. 1. Proteome array of conditioned medium derived from HSC-Li cells. Conditioned medium derived from HSC-Li cells was examined with proteome profiler human arrays specific for cytokines and growth factors. Cytokines can be divided into six classes: hepatocyte protection, hepatocyte growth, anti-apoptosis, liver-specific metabolic activity enhancement, anti-inflammation, and angiogenesis. The horizontal axis represents the expression levels of cytokines in conditioned medium normalized to that in serum-free DMEM.
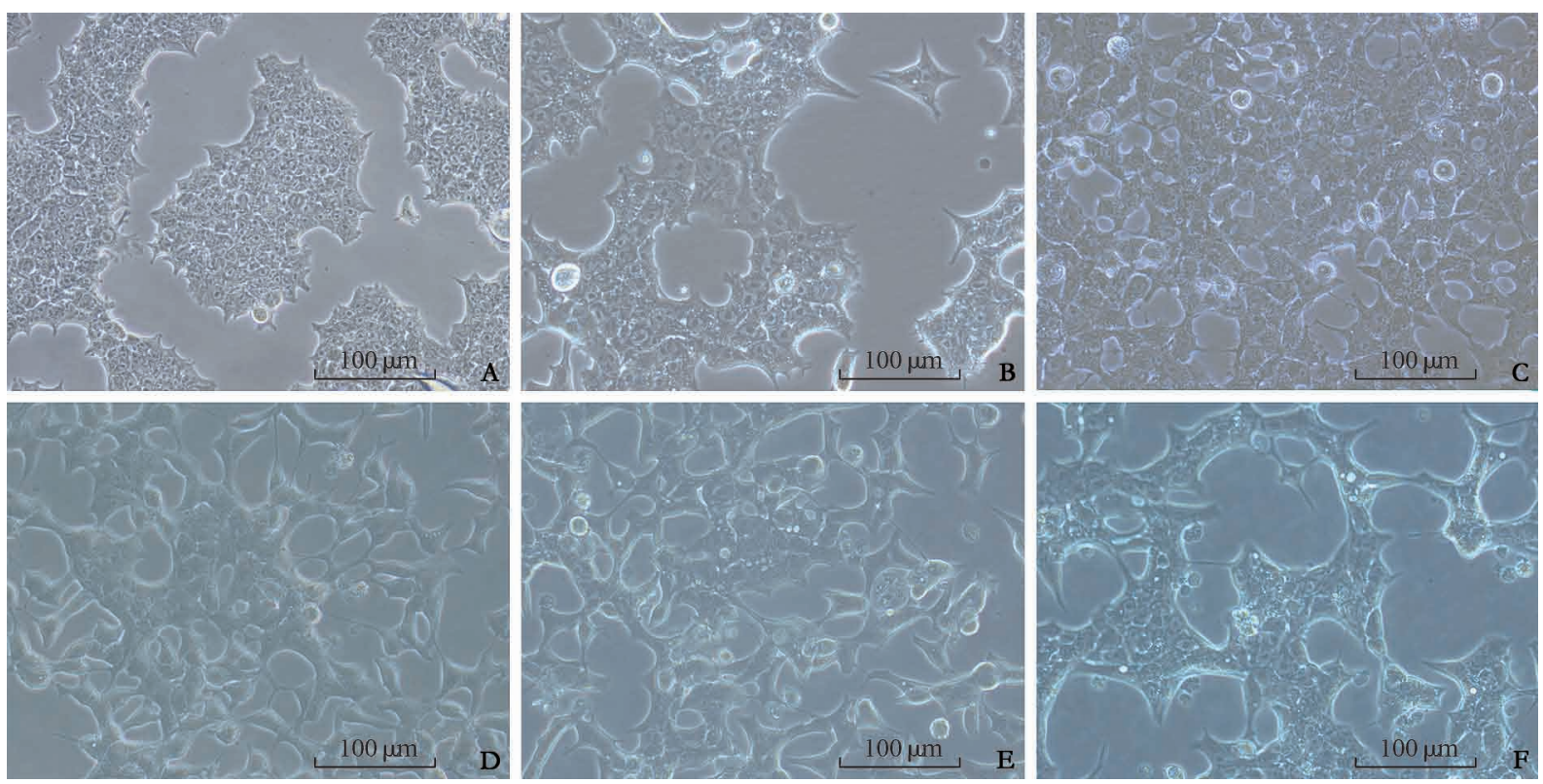

Fig. 2. Morphological features of mouse LPC-derived cells. A: Mono-cultured BMEL-TAT cells were observed under an optical microscope after 1 day. Initial small cells were phenotypically immature with scant cytoplasm and oval-shaped nuclei. B-F: Representative morphology of BMEL-TAT cells were recorded at days 1, 3, 7, 14, and 21 in co-culture process. Scale bar: $100 \mu \mathrm{m}$. 

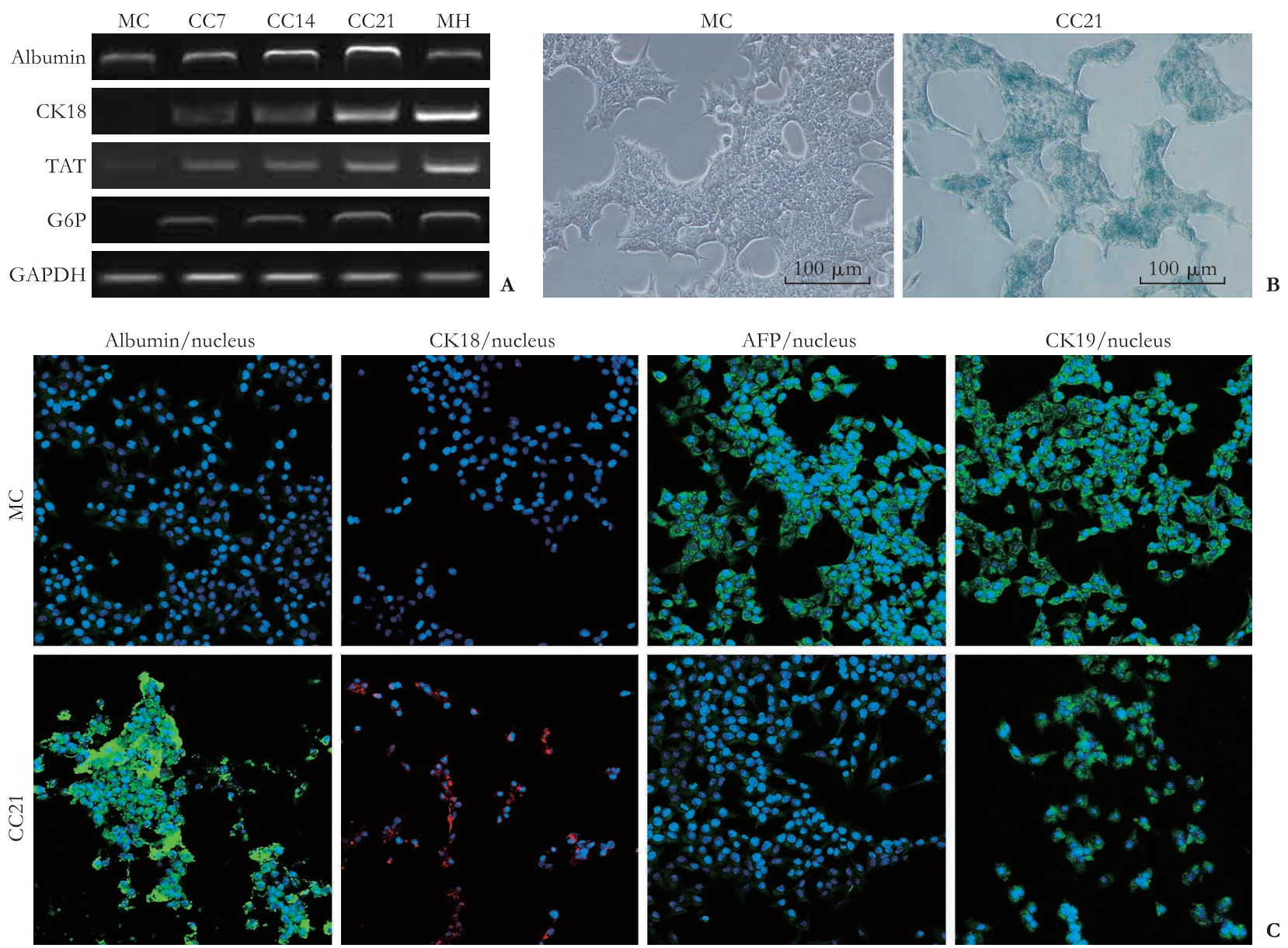

Fig. 3. Characteristics of mouse LPC-derived cells. A: The mRNA expression levels of albumin, CK18, TAT, and G6P were gradually upregulated over the course of the differentiation process. MC stood for control (mono-culture for 1 day), CC7 for co-culture after 7 days, CC14 for co-culture after 14 days, CC21 for co-culture after 21 days, and $\mathrm{MH}$ for positive control (primary mouse hepatocytes). B: X-gal staining was strongly positive in BMEL-TAT cells after 21-day co-culture with HSC-Li cells. The mono-cultured BMEL-TAT cells were regarded as negative controls. Scale bar: $100 \mu \mathrm{m}$. C: Fluorescence of albumin and CK18 strengthened while that of AFP and CK19 weakened in co-cultured BMEL-TAT cells after 21 days, compared with mono-cultured BMEL-TAT cells. Cells were costained with DAPI to identify nuclei.

(Fig. 3B). The abundance of hepatocyte-specific proteins (albumin and CK18) increased while that of immature proteins (AFP and CK19) decreased, which correlated with the levels of mRNA detected by PCR (Fig. 3C). Collectively, these results suggested that HSC-Li cells induce the differentiation of LPCs into hepatocyte-like cells.

\section{Characterization of differentiated LPCs with respect to hepatocyte function}

The differentiated cells exhibited strong PAS staining (Fig. 4A), reflecting their ability to store glycogen. By contrast, conventionally cultured BMEL-TAT cells were negative. Compared with mono-cultured BMEL-TAT cells, the differentiated cells take up more LDL (Fig. 4B). They also secrete albumin, metabolize drugs (P450, CYP1A2 activity), and synthesize urea. Albumin secretion, CYP1A2, and urea synthesis were significantly enhanced (Fig. 4C-E). These results showed that HSC-Li cells fur- ther matured already differentiated LPCs.

\section{Discussion}

In this study, we have defined an in vitro hepatic differentiation protocol, i.e., an indirect co-culture system with immortalized HSCs, which can efficiently and reproducibly induce the differentiation of mouse LPCs into functional hepatocyte-like cells that possess many liver characteristics and functions.

Recognizing the importance of interactions between hepatic stem cells and hepatic nonparenchymal cells in liver development, we co-cultured LPCs with immortalized human HSCs to assess their ability to enhance the differentiation toward functional hepatocytes. The nontumorigenic human HSC-Li is an activated HSC based on its expression of $\alpha$-SMA and production of extracellular matrix. HSC-Li cells are potentially useful as a feed- 

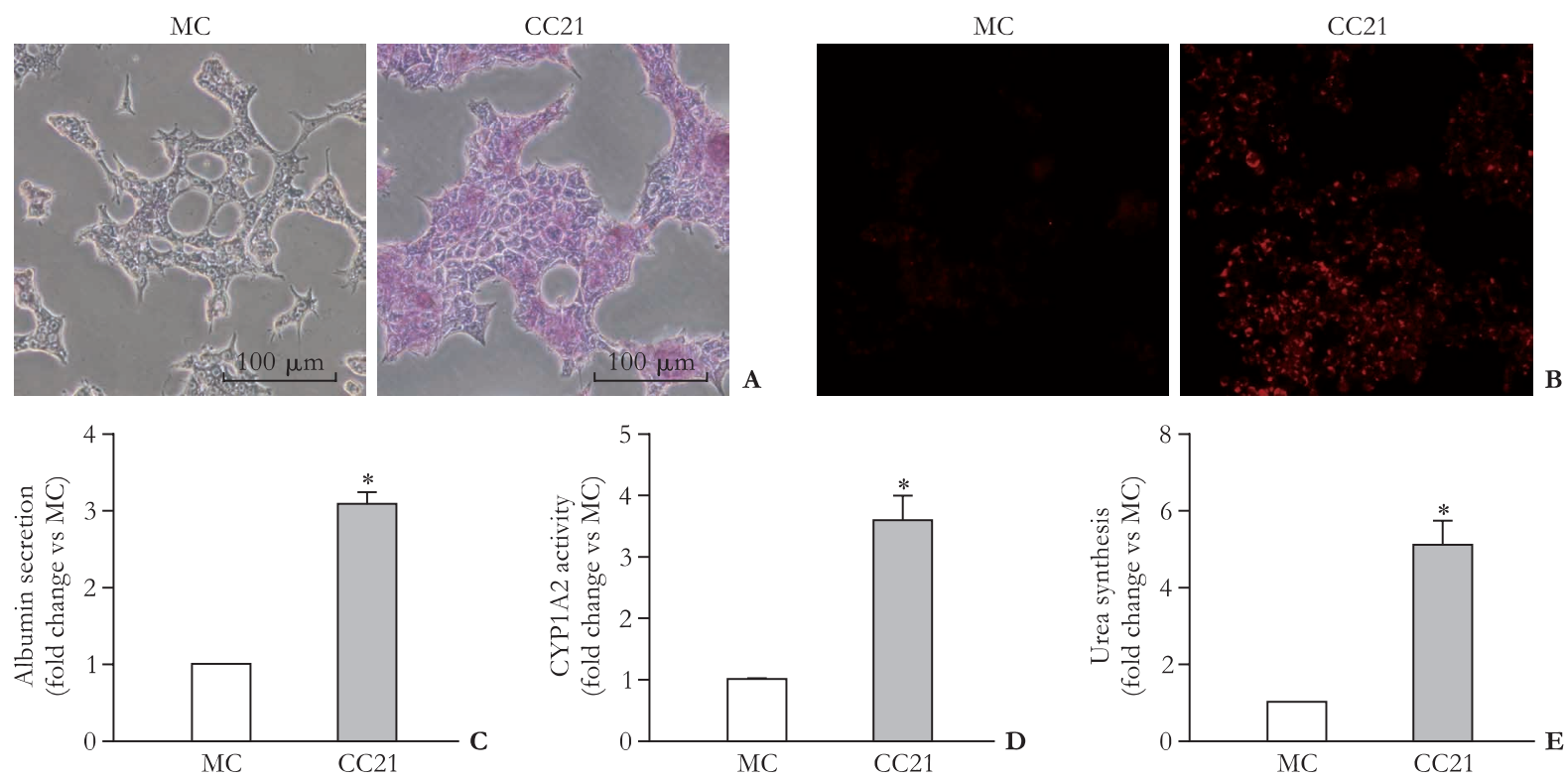

Fig. 4. Hepatocyte functional analysis of mouse LPC-derived cells. PAS staining was absent in mono-cultures (MC) and strongly positive in co-cultures (CC21) of BMEL-TAT cells with HSC-Li cells after 21-day co-culture (A). LDL endocytosis was absent in monocultures (MC) and strongly positive in co-cultures (CC21) of BMEL-TAT cells with HSC-Li cells after 21-day co-culture (B). Secretion of albumin (C), CYP1A2 activity (D), and urea synthesis (E) were substantially higher in co-cultured (CC21) than mono-cultured (MC) BMEL-TAT cells. Data are expressed as mean \pm SD relative units. Scale bar: $100 \mu \mathrm{m}$. *: $P<0.05$, vs MC.

er layer that promotes hepatic differentiation because it produces growth factors and cytokines that influence LPCs, such as HGF, EGF, VEGF-D, IL-6sR, and TNF- $\alpha$. All these factors are important for liver development and regeneration. ${ }^{[3]}$ HGF plays an essential role as an endocrine or paracrine factor in hepatocyte differentiation and induces the differentiation of stem/progenitor cells in vitro. ${ }^{[4,8,9]}$ The present study demonstrated that our co-culture system of LPCs with HSC-Li efficiently induces hepatic differentiation, which is in agreement with the findings of previous studies that HSCs are involved in the differentiation of hepatic stem cells by paracrine secretion. ${ }^{[21,22]}$

The differentiation of LPCs showed changes in morphology to hepatocytes and the expression of liverenriched genes, namely, albumin, CK18, TAT, and G6P. We found that the expression of albumin after 14 days of co-culture was higher than that of primary hepatocytes. This finding suggested that HSC-Li cells strongly induce the differentiation of LPCs into hepatocyte-like cells. Although the differentiated cells displayed features of mature hepatocytes, some nonhepatic markers also persisted. Along with the strong albumin expression, immunofluorescence staining revealed that a small population of differentiated cells still expressed AFP, indicating that they have not acquired a fully mature hepatocyte phenotype. During the development of the human liver, one of the first antigens detected in progenitor cells is CK19, which disappears when progenitor cells differentiate into hepatocytes. By contrast, an increasing expression of CK19 can be observed when cells commit toward bile duct cell lineage. ${ }^{[28]}$ In the present study, the majority of differentiated cells still expressed CK19, suggesting that they could still be capable of generating cholangiocytes. Collectively, the data suggest that a population comprising both mature and immature hepatocytes, possibly LPCs makes up the differentiated culture, this may be an advantage, as the LPCs could continue to generate immature and mature hepatocytes. This study also showed that the co-culture system produced hepatocyte-like cells from LPCs that acquired other important functions. These include glycogen storage, LDL uptake, albumin secretion, and urea synthesis.

In conclusion, we represent a simple and reproducible method to induce the differentiation of mouse LPCs into functional hepatocyte-like cells by indirect co-culture with immortalized HSCs. This novel method provides a novel approach to differentiate LPCs and an important step in the overall strategy for cell therapy of liver pathologies. It also raises possibilities for using these cells to screen agents or drugs that induce cytotoxicity.

Contributors: PXP, YGC and LLJ conceived and designed the study. PXP, WYN, YXP, ZCX and LYZ performed the research. PXP and WYN analyzed the data. PXP, WYN and YGC wrote and revised the article. PXP and WYN equally contributed to the article. All authors contributed to the design and interpretation of the study and to further drafts. YGC and LLJ are the guarantors.

Funding: This study was supported by grants from the Chinese 
High-Tech Research \& Development (863) Program (2013AA020102 and 2012AA020204), Science Fund for Creative Research Groups of the National Natural Science Foundation of China (81121002), Fundamental Research Funds for the Central Universities (2014XZZX008 and 2014FZA7010), Zhejiang CTM Science and Technology Project (2011ZB061), Zhejiang Health Science Foundation (2016KYA148), and the National Health and Medical Research Council of Australia and Cancer Council of Western Australia.

Ethical approval: All animal experiments were performed in accordance with the animal guidelines of Zhejiang University School of Medicine.

Competing interest: No benefits in any form have been received or will be received from a commercial party related directly or indirectly to the subject of this article.

\section{References}

1 Basma H, Soto-Gutiérrez A, Yannam GR, Liu L, Ito R, Yamamoto $\mathrm{T}$, et al. Differentiation and transplantation of human embryonic stem cell-derived hepatocytes. Gastroenterology 2009;136:990-999.

2 Stock P, Brückner S, Winkler S, Dollinger MM, Christ B. Human bone marrow mesenchymal stem cell-derived hepatocytes improve the mouse liver after acute acetaminophen intoxication by preventing progress of injury. Int J Mol Sci 2014;15: 7004-7028.

3 Soto-Gutiérrez A, Kobayashi N, Rivas-Carrillo JD, NavarroAlvarez N, Zhao D, Okitsu T, et al. Reversal of mouse hepatic failure using an implanted liver-assist device containing ES cell-derived hepatocytes. Nat Biotechnol 2006;24:1412-1419.

4 Roelandt P, Sancho-Bru P, Pauwelyn K, Verfaillie C. Differentiation of rat multipotent adult progenitor cells to functional hepatocyte-like cells by mimicking embryonic liver development. Nat Protoc 2010;5:1324-1336.

5 Salomone F, Barbagallo I, Puzzo L, Piazza C, Li Volti G. Efficacy of adipose tissue-mesenchymal stem cell transplantation in rats with acetaminophen liver injury. Stem Cell Res 2013;11: 1037-1044.

6 Okabe M, Tsukahara Y, Tanaka M, Suzuki K, Saito S, Kamiya Y, et al. Potential hepatic stem cells reside in EpCAM+ cells of normal and injured mouse liver. Development 2009;136:1951-1960.

7 Shin S, Walton G, Aoki R, Brondell K, Schug J, Fox A, et al. Foxl1-Cre-marked adult hepatic progenitors have clonogenic and bilineage differentiation potential. Genes Dev 2011;25: 1185-1192.

8 Zhang W, Li W, Liu B, Wang P, Li W, Zhang H. Efficient generation of functional hepatocyte-like cells from human fetal hepatic progenitor cells in vitro. J Cell Physiol 2012;227:2051-2058.

9 Chen Y, Zhou H, Sarver AL, Zeng Y, Roy-Chowdhury J, Steer CJ, et al. Hepatic differentiation of liver-derived progenitor cells and their characterization by microRNA analysis. Liver Transpl 2010;16:1086-1097.

10 Iacob R, Rüdrich U, Rothe M, Kirsch S, Maasoumy B, Narain $\mathrm{N}$, et al. Induction of a mature hepatocyte phenotype in adult liver derived progenitor cells by ectopic expression of transcription factors. Stem Cell Res 2011;6:251-261.

11 Suetsugu A, Nagaki M, Aoki H, Motohashi T, Kunisada T, Moriwaki H. Differentiation of mouse hepatic progenitor cells induced by hepatocyte nuclear factor- 4 and cell transplantation in mice with liver fibrosis. Transplantation 2008;86:1178-1186.
12 Blanpain C, Lowry WE, Geoghegan A, Polak L, Fuchs E. Selfrenewal, multipotency, and the existence of two cell populations within an epithelial stem cell niche. Cell 2004;118:635-648.

13 Enver T, Greaves M. Loops, lineage, and leukemia. Cell 1998; 94:9-12.

14 Jori FP, Galderisi U, Piegari E, Cipollaro M, Cascino A, Peluso G, et al. EGF-responsive rat neural stem cells: molecular followup of neuron and astrocyte differentiation in vitro. J Cell Physiol 2003;195:220-233.

15 Quesenberry PJ, Becker PS. Stem cell homing: rolling, crawling, and nesting. Proc Natl Acad Sci U S A 1998;95:15155-15157.

16 Urbanek K, Cesselli D, Rota M, Nascimbene A, De Angelis A, Hosoda T, et al. Stem cell niches in the adult mouse heart. Proc Natl Acad Sci U S A 2006;103:9226-9231.

17 Wang Y, Yao HL, Cui CB, Wauthier E, Barbier C, Costello MJ, et al. Paracrine signals from mesenchymal cell populations govern the expansion and differentiation of human hepatic stem cells to adult liver fates. Hepatology 2010;52:1443-1454.

18 Tuleuova N, Lee JY, Lee J, Ramanculov E, Zern MA, Revzin A. Using growth factor arrays and micropatterned co-cultures to induce hepatic differentiation of embryonic stem cells. Biomaterials 2010;31:9221-9231.

19 Yu YD, Kim KH, Lee SG, Choi SY, Kim YC, Byun KS, et al. Hepatic differentiation from human embryonic stem cells using stromal cells. J Surg Res 2011;170:e253-261.

20 Nagamoto Y, Tashiro K, Takayama K, Ohashi K, Kawabata K, Sakurai F, et al. The promotion of hepatic maturation of human pluripotent stem cells in $3 \mathrm{D}$ co-culture using type I collagen and Swiss 3T3 cell sheets. Biomaterials 2012;33:4526-4534.

21 Deng X, Chen YX, Zhang X, Zhang JP, Yin C, Yue HY, et al. Hepatic stellate cells modulate the differentiation of bone marrow mesenchymal stem cells into hepatocyte-like cells. J Cell Physiol 2008;217:138-144.

22 Nagai H, Terada K, Watanabe G, Ueno Y, Aiba N, Shibuya T, et al. Differentiation of liver epithelial (stem-like) cells into hepatocytes induced by coculture with hepatic stellate cells. Biochem Biophys Res Commun 2002;293:1420-1425.

23 Pan X, Wang Y, Yu X, Li J, Zhou N, Du W, et al. Establishment and characterization of an immortalized human hepatic stellate cell line for applications in co-culturing with immortalized human hepatocytes. Int J Med Sci 2015;12:248-255.

24 Strick-Marchand H, Weiss MC. Inducible differentiation and morphogenesis of bipotential liver cell lines from wild-type mouse embryos. Hepatology 2002;36:794-804.

25 Tirnitz-Parker JE, Tonkin JN, Knight B, Olynyk JK, Yeoh GC. Isolation, culture and immortalisation of hepatic oval cells from adult mice fed a choline-deficient, ethionine-supplemented diet. Int J Biochem Cell Biol 2007;39:2226-2239.

26 Kobayashi N, Ito M, Nakamura J, Cai J, Gao C, Hammel JM, et al. Hepatocyte transplantation in rats with decompensated cirrhosis. Hepatology 2000;31:851-857.

27 Yang Y, Li J, Pan X, Zhou P, Yu X, Cao H, et al. Co-culture with mesenchymal stem cells enhances metabolic functions of liver cells in bioartificial liver system. Biotechnol Bioeng 2013;110: 958-968.

28 Haruna Y, Saito K, Spaulding S, Nalesnik MA, Gerber MA. Identification of bipotential progenitor cells in human liver development. Hepatology 1996;23:476-481.

Received June 28, 2015 Accepted after revision January 29, 2016 